\title{
Assembly and disassembly of viral capsids
}

\author{
ROYA ZANDI $\dagger *$, DAVID REGUERA $\dagger$, ROBIJN BRUINSMA $\ddagger$ WILLIAM GELBART $\dagger$ and JOSEPH RUDNICK $\ddagger$
}

$\dagger$ Department of Chemistry, University of California, Los Angeles, LA 90095, USA

¥Department of Physics, University of California, Los Angeles, LA 90095, USA

\begin{abstract}
We address here a theoretical basis for the icosahedral symmetry that is observed so commonly for viral capsids, i.e., the single-protein-thick rigid shells that protect the viral genome. In particular, we outline the phenomenological hamiltonian approach developed recently (see Zandi, R., Reguera, D., Bruinsma, R., Gelbart, W.M. and Rudnick, J. (2004), Original of icosahedral symmetry in viruses, Proc. Natl. Acad. Sci., 101, 15556-15560) to account for the overwhelming prevalence of the Caspar-Klug " $T$ number" structures that are found for "spherical" viruses. We feature the role of "conformational switching energies" defining the competing multimeric states of the protein subunits. The results of Monte Carlo simulation of this model are argued to shed light as well on the mechanical properties and genome release mechanism for these viruses.
\end{abstract}

Keywords: Viral self-assembly; Icosahedral symmetry; Structural transitions; $T$-number

\section{Introduction}

The cowpea rotic mottle virus (CCMV) is a classical $T=3$ RNA virus with a shell composed of 180 identical subunits, [2] see also A. Zlotnick, this volume. Figure 1(a) is cryo-TEM image showing 5- and 6-fold morphological units while figure $1(b)$ shows the associated arrangement of the subunits. The capsid has 12 five-fold symmetry sites, 20 three-fold symmetry sites and 30 two-fold symmetry sites. The subunits occupy three different types of subunit locations, denoted by A, B and C, that do not transform into each other under the symmetry operations. The subunits can be divided into 12 capsomers that contain 5 subunits ("pentamers") and 20 capsomers that contain 6 subunits ("hexamers"). This architecture presumably represents a free minimum because CCMV reversibly self-assembles from its molecular components under in vitro conditions [3]. More generally, Caspar and Klug (CK) [2] showed that the subunits of icosahedral shells constructed from pentamers and hexamers occupy $T$ non-equivalent locations, with the $T$-number adopting the values $1,3,4,7,13, \ldots$. A CK icosahedral shell always contains 12 pentamers plus $10(T-1)$ hexamers (so $60 T$ subunits in total). This CK classification is the foundation of modern structural virology. It minimizes the number of symmetry non-equivalent subunit contacts (known as the "quasi-equivalence" principle) and, on this basis, is believed to describe the generic structure of protein shells. ${ }^{\S}$ However, with current computational capabilities it is neither possible to verify this claim through all-atom molecular dynamics simulations nor can we design artificial protein shells by all-atom simulations.

We recently investigated a simplified model [1] for the assembly of molecular shells based on capsomer units that can switch between two internal states $\mathrm{P}$ and $\mathrm{H}$, which can be viewed-but not necessarily—as pentamers and hexamers (capsid models without internal degrees of freedom do not reproduce icosahedral symmetry, as discussed below). $\mathrm{P}$ and $\mathrm{H}$ capsomer differ only in size, with a radially symmetric capsomer-capsomer potential. The number of $\mathrm{P}$ units is free to vary and is not fixed to be 12 (as in the CK construction). The energy difference $\Delta E$ between a $\mathrm{P}$ and an $\mathrm{H}$ subunit enters as a Boltzmann probability factor $e^{-\Delta E / k_{B} T}$ for units to be in the P state. During a simulation run, $N$ interacting capsomers are allowed to range over a spherical surface starting from a random initial configuration. The free energy $F(R)$ is evaluated for a range of sphere radii $R$ and minimized with respect to $R$. The capsomer-capsomer interaction potential $V(r)$ depends only on the separation $r$ between the capsomer centres. It consists of a short-range repulsion, representing subunit conformational rigidity, plus a longer-range attraction, representing capsomercapsomer attraction (e.g. by hydrophobic interaction).

*Corresponding author: Tel.: +1-310-825-6790. Fax: +1-310-206-4038. Email: roya @ physics.ucla.edu

I Tel.: + 1-310-825-8539. Fax: + 1-310-206-5668. Email: bruinsma@ physics.ucla.edu.

${ }^{\S}$ The CK classification applies as well to viral shells that do not show a pentamer/hexamer organization. 
(a)

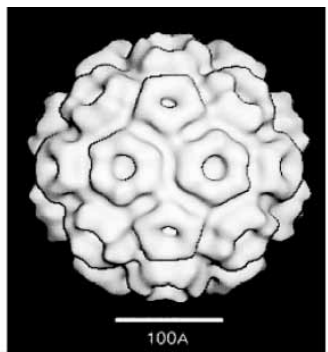

(b)

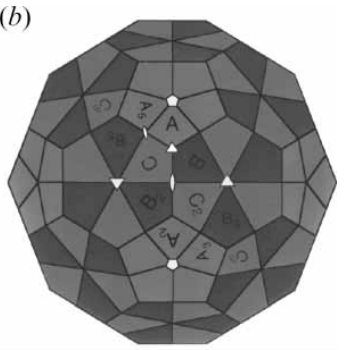

Figure 1. (a) Cryo-TEM image of CCMV. (b) Arrangement of subunits on a truncated icosahedron. $\mathrm{A}, \mathrm{B}$ and $\mathrm{C}$ stand for symmetry nonequivalent sites.

The capsomer-capsomer binding energy $\varepsilon_{0}$ was taken to be $15 k_{\mathrm{B}} T$, a typical value reported by all-atom calculations of subunit binding energies [4]. The $V(r)$ potential had the same form for interactions between different capsomer types except that the equilibrium spacing-the minimum of $V(r)$-was adjusted to include a size difference between $\mathrm{P}$ and $\mathrm{H}$ capsomers [5]. The $\mathrm{P} / \mathrm{H}$ size ratio was taken to be 0.85 , appropriate for equilateral pentagons and hexagons of the same edge length (see figure $1(b)$ ). The simplicity of the interaction potential was motivated by the fact that the design rules for viral capsids hold for subunits having different folding domains and polypeptide sequences [6]. We tested different forms for $V(r)$ and found our results to be robust.

\section{Monte-Carlo simulations}

The results of the Monte Carlo simulations are shown in figure 2 as a plot of the free energy per capsomer $\varepsilon(N)$, expressed in units of $\varepsilon_{0}$ versus the number $N$ of capsomers. The black solid line shows $\varepsilon(N)$ for the case that the energy difference $\Delta E$ between $\mathrm{P}$ and $\mathrm{H}$ states equals zero. For larger $N$ values, $\varepsilon(N)$ is slightly less than $-3 \varepsilon_{0}$, the binding energy per capsomer for a flat hexagonal array of capsomers. Pronounced minima of $\varepsilon(N)$ are found at $N=72,42,32$ and 12. The structures are shown in figure 3(a), with the capsomers shown, for simplicity, as disks. All four minimal structures have icosahedral symmetry and correspond to, respectively, the $T=7$, $T=4, T=3$, and $T=1 \mathrm{CK}$ structures (the $N=32 / T=3$ structure should be compared with figure $1(b)$ ). In all four cases, the equilibrium configuration developed spontaneously with no evidence for kinetic traps, though the $T=7$ chiral repeat motif is already fairly complex.

Capsid self-assembly commences when the solution chemical potential of isolated capsomers exceeds $-\varepsilon(N)$ [7]. Actual size selection of viruses involves certain additional mechanisms that vary between viral species, such as "spontaneous curvature" effects [8], the presence of preformed scaffold structures [10], or the size of the enclosed genome. Assume that these effects restrict the capsid size to a range of $N$ values, say $N=70 \pm 10$, that includes one of the minima of $\varepsilon(N)(N=72$ in this case). According to figure 2 , the energy per capsomer of the $N=72$ capsid is only about $0.05 \varepsilon_{0}$ less than that of its neighbours, $N=71$ and $N=73$, but the total energy difference ( 72 times larger) is about $55 k_{\mathrm{B}} T$. The relative abundance of capsids with $N \neq 72$ depends on the total energy difference as $e^{-(N \varepsilon(N)-72 \varepsilon(72)) / k_{B} T}$, so the relative abundance of the $N=71$ and $N=73$ structures is completely negligible. Thus, because the minimum of $\varepsilon(N)$ at $N=72$ is sufficiently pronounced, additional size-determining mechanisms need only to provide a weak dependence on $N$ to produce a nearly monodisperse solution of $T=7$ capsids during selfassembly.

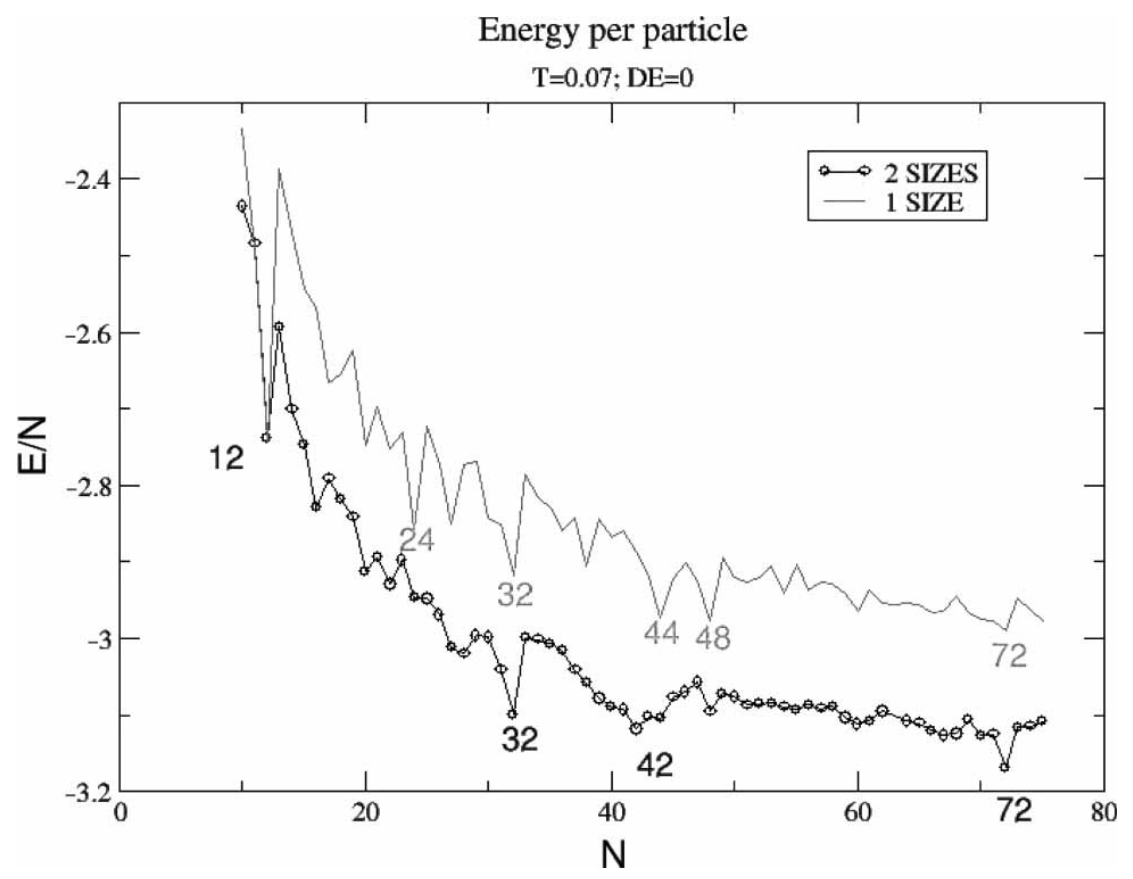

Figure 2. Energy per capsomer for $\Delta E=0$ (open circles), and for $\left|\Delta E / k_{\mathrm{B}} T\right|$ large compared to one (solid line). 

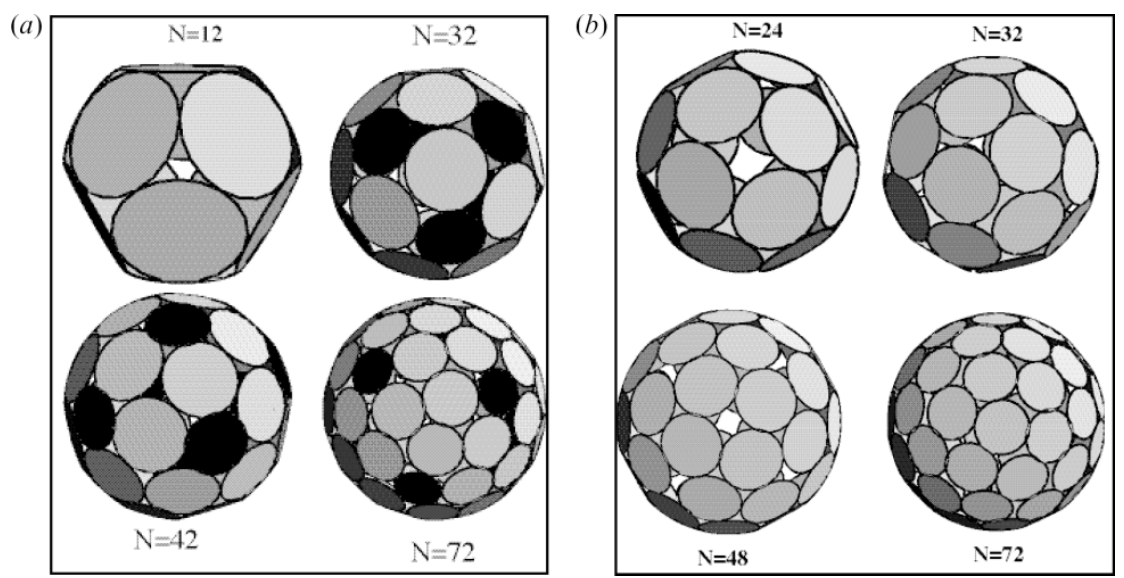

Figure 3. Minimum free energy structures. (a) The $\mathrm{P}$ and $\mathrm{H}$ states are degenerate. The $N=12,32,42$ and 72 structures correspond to stable $T=1,3,4,7$ icosahedra, respectively. P state capsomers are shown in black. For clarity the capsomer size was scaled, so all capsids have the same size. (b) The P state energy is large compared to the capsomer binding energy. $N=24$ and 48 have octahedral symmetry. $N=32$ corresponds to $T=3$. The $N=72$ structure is thermally unstable.

(a)

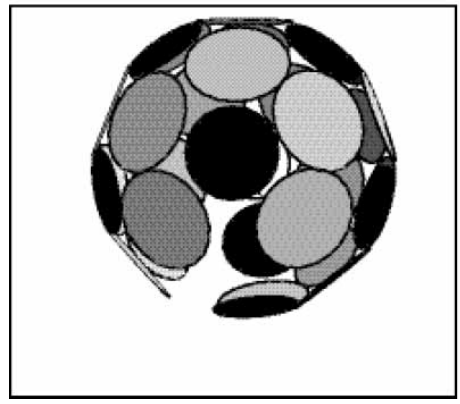

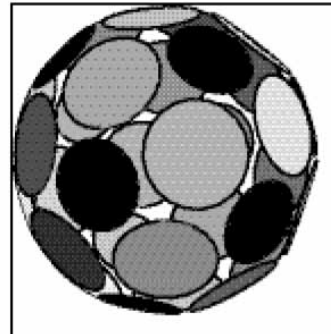

(a) (b)

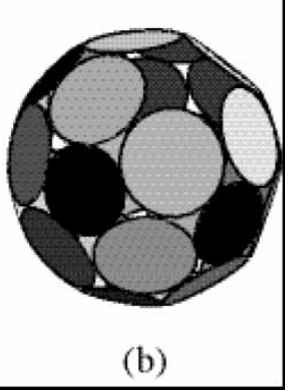

Figure 4. Capsid disassembly. (a) Bursting of a $T=3$ capsid under internal pressure when the ratio $R / R^{*}$ of the capsid radius and the equilibrium radius exceeds 1.107. (b) Capsid just before $(a)$ and just after $(b)$ pentamer decapsidation $\left(R / R^{*}=1.013\right)$.

When we increase the P-to-H switch energy $\Delta E / k_{\mathrm{B}} T$, we encounter a dramatic reorganization of the structure spectrum for $\Delta E$ values around $1-2 \varepsilon_{0}$. The green curve in figure 2 gives $\varepsilon(N)$ for large $\left|\Delta E / \varepsilon_{0}\right|$. The units now are either all in the $\mathrm{H}$ state or all in the $\mathrm{P}$ state, depending on the sign of $\Delta E$. Pronounced minima in $\varepsilon(N)$ are seen at $N=12,24,32,44,48$ and a weak minimum at 72 . The corresponding capsids are shown in figure $3(b)$. The new sequence of "magical numbers" can be understood by considering the packing of $N$ hard disks on a spherical surface, known in the mathematics literature as the Tammes Problem. The magical numbers that minimize $\varepsilon(N)$ correspond to the maxima of the packing density of the Tammes Problem [9].

The suppression of capsomer switching clearly has profoundly destabilizing effects on icosahedral shells. The only surviving $T$-structures are $T=1(N=12)$ and $T=3$ $(N=24)$. The new $N=24$ and 48 minima have octahedral symmetry, while $N=44$ has cubic symmetry [5]. Of particular interest is the $N=24$ case, a chiral octahedral
Archimedean solid known as the "snub-cube" (see figure $3(b)$ ). Self-assembly studies of Polyoma capsid proteins - an exceptional virus whose capsomers $d o$ all have the same size - report the formation of stable $N=24$ capsids with the symmetry of a left-handed snub-cube [11]. The $N=72$ structure is unstable: it undergoes dramatic thermal fluctuations between different structures of various symmetry, including $T=7$. Moreover, self-assembly at larger $N$ values is predicted to produce a polydisperse mixture of capsid sizes.

\section{Capsid disassembly}

The enclosed genome of a virus applies a significant osmotic pressure on the viral shell. This pressure and the associated tension of the capsid wall play an important role during genome release [12]. Osmotic pressure $\Pi$ can be included in our simulation by imposing capsid radii $R$ different from the equilibrium radius $R^{*}$ through $\Pi(R) \propto\left(R / R^{*}-1\right) .^{\dagger}$ We encountered the following

${ }^{\dagger}$ Specifically, with $\Pi(R)=\Pi_{0}(N)\left(R / R^{*}-1\right)$ with $\Pi_{0}(N)=\left.\left(N /\left(4 \pi R^{*}\right)\right)\left(\left(\mathrm{d}^{2} \varepsilon(R)\right) / \mathrm{d} R^{2}\right)\right|_{R=R^{*}}$. For the present case with $N=32$ and $R^{*}=10 \mathrm{~nm}$, we find $\Pi_{0}(32) \approx 7 \mathrm{~atm}$ 
sequence of events for $N=32(T=3)$ when we increased $R$ beyond $R^{*}$. If we keep both the numbers of $\mathrm{P}$ and $\mathrm{H}$ capsomers fixed, then the capsid bursts in a dramatic manner after $R / R^{*}$ exceeds a critical value of 1.107 (see figure 4(a)). Capsid bursting under external osmotic shock is a well-known phenomenon that can be used to measure the mechanical strength of capsids [13].

If the capsomer number $N$ is allowed to change, then the capsid pressure can be released before bursting. The energy of a $T=3(N=32)$ capsid exceeds that of an $N=31$ icoasahedral capsid with one pentamer removed from one of the 5-fold sites (see figure 4(b)) when $R / R^{*}$ exceeds 1.013 . Decapsidation by pentamer release is, in fact, the established genome release mechanism for Tymoviruses [14]. Genome release scenarios through pentamer conformational changes have been proposed for the picorna and nodaviruses [15]. We studied pressure-induced conformational changes by permitting conversion between $\mathrm{P}$ to $\mathrm{H}$ capsomers at fixed $N$. We found that $\mathrm{P}$ capsomers progressively transform to $\mathrm{H}$ capsomers, with loss of icosahedral symmetry. These studies suggest that our two-state model can be usefully applied to further investigation of the mechanical and structural properties of viral capsids.

\section{References}

[1] Zandi, R., Reguera, D., Bruinsma, R., Gelbart, W.M. and Rudnick, J., 2004, Origin of icosahedral symmetry in viruses. Proc. Natl. Acad. Sci., 101, 15556-15560.

[2] Caspar, D.L.D. and Klug, A., 1962, Physical principles in the construction of regular viruses. Cold Spring Harbor Symp. Quant. Biol., 27, 1-22.
[3] Adolph, K.W. and Butler, J., 1976, Assembly of a spherical plant virus. Philos. Trans. R. Soc. Lond. B Biol. Sci., 276, 113-122.

[4] Reddy, V.S., Giesing, H.A., Morton, R.T., Kumar, A., Post, C.B., Brooks, C.L., III and Johnson, J.E., 1998, Energetics of quasie quivalence: computational analysis of protein-protein interactions in icosahedral viruses. Biophys. J., 74, 546-558.

[5] Voogd, J., Crystalization on a sphere: Computational studies of twodimensional Lennard-Jones systems. Thesis, Universiteit van Amsterdam, 1998; Specifically: $V(r)=\varepsilon_{0}\left[\left(r^{*} / r\right)^{12}-2\left(r^{*} / r\right)^{6}\right]$ with $\varepsilon_{0}$ the pair binding energy and $r^{*}$ the equilibrium spacing. For an earlier study with one-state units:

[6] Brandon, C. and Tooze, J., 1999, Ch.16. Introduction to Protein Structure, 2nd Ed. (New York: Garland).

[7] Bruinsma, R.F., Gelbart, W.M., Reguera, D., Rudnick, J. and Zandi, R., 2003, Viral self-assembly as a thermodynamic process. Phys. Rev. Lett., 90, 248101.

[8] Johnson, J.E. and Speir, J.A., 1997, Quasi-equivalent viruses: a paradigm for protein assemblies. J. Mol. Biol., 269, 665-675.

[9] Clare, B.W. and Keppert, D.L., 1986, The closest packing of equal circles on a sphere. Proc. R. Soc. Lond. A, 405, 329-344.

[10] Thuman-Commike, P.A., Greene, B., Malinski, J.A., King, J. and Chiu, W., 1998, Role of the scaffolding protein in P22 procapsid size determination Suggested by $\mathrm{T}=4$ and $\mathrm{T}=7$ procapsid structures. Biophys. J., 74, pp. 559-568.

[11] Salunke, D.M., Caspar, D.L. and Garcea, R.L., 1989, Polymorphism in the assembly of polyomavirus capsid protein VP1. Biophys. J., 56, 887-900.

[12] Tzlil, S., Kindt, J.T., Gelbart, W.M. and Ben-Shaul, A., 2003, Forces and pressures in DNA packaging and release from viral capsids. Biophys. J., 84, 1616-1627. Capsid pressure $\Pi$ and capsid tension $\tau$ are related by $\Pi=2 \tau / R$.

[13] Cordova, A., Deserno, M., Gelbart, W.M. and Ben-Shaul, A., 2003, Osmotic shock and the strength of viral capsids. Biophys. J., 85, 70-74.

[14] Adrian, M., Timmins, P.A. and Witz, J., 1992, In vitro decapsidation of turnip yellow mosaic virus investigated by cryo-electron microscopy: A model for the decapsidation of a small isometric virus. J. Gen. Virol., 73, 2079-2083, Capsid tension of Tymoviruses is believed to be $\mathrm{pH}$ sensitive.

[15] Johnson, J.E., 1996, Functional implications of protein-protein interactions in icosahedral viruses. Proc. Natl Acad. Sci., 93, $27-33$. 


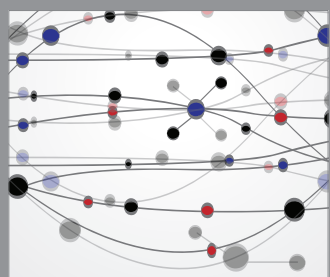

The Scientific World Journal
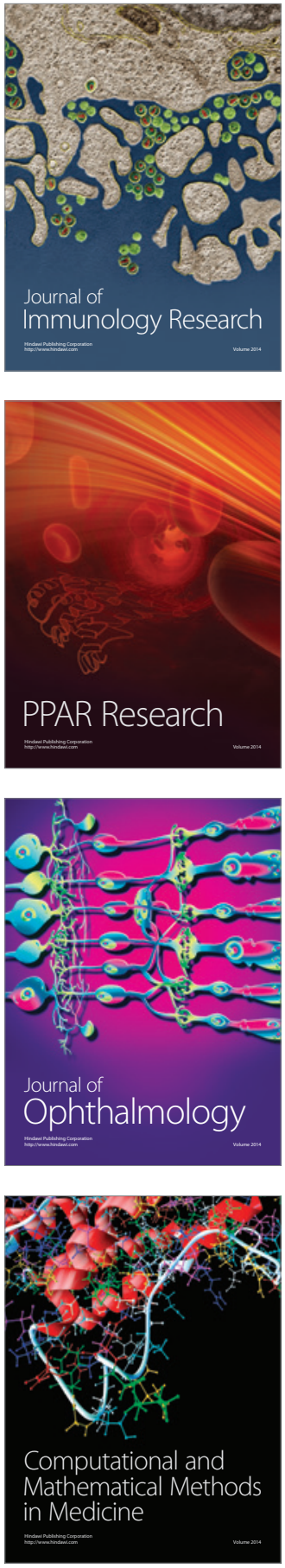

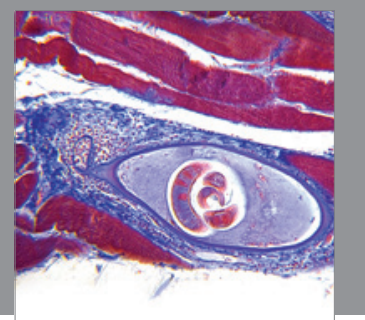

Gastroenterology

Research and Practice
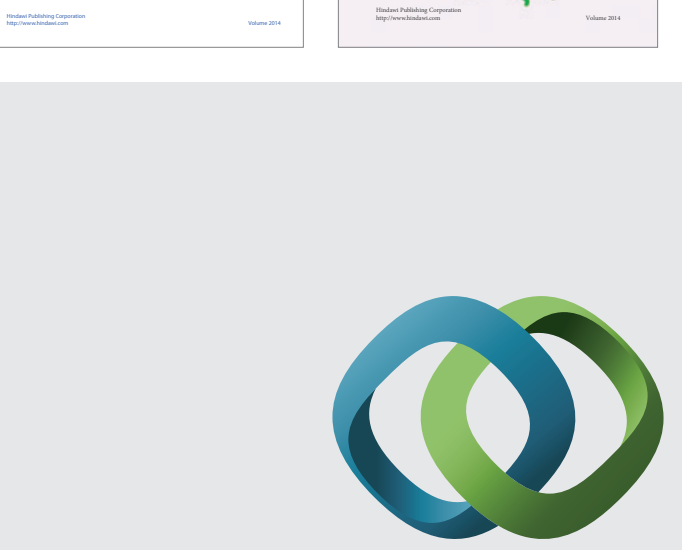

\section{Hindawi}

Submit your manuscripts at

http://www.hindawi.com
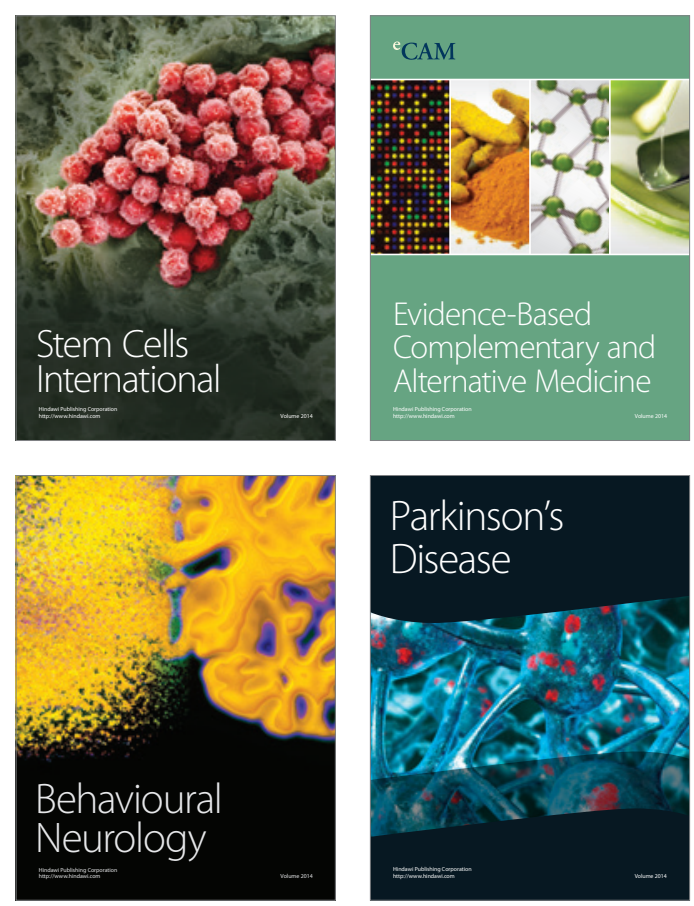

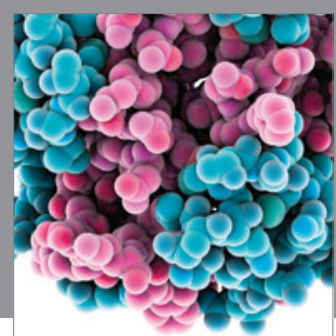

Journal of
Diabetes Research

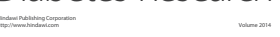

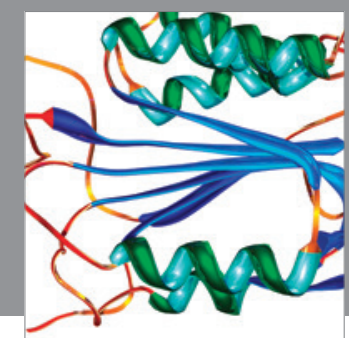

Disease Markers
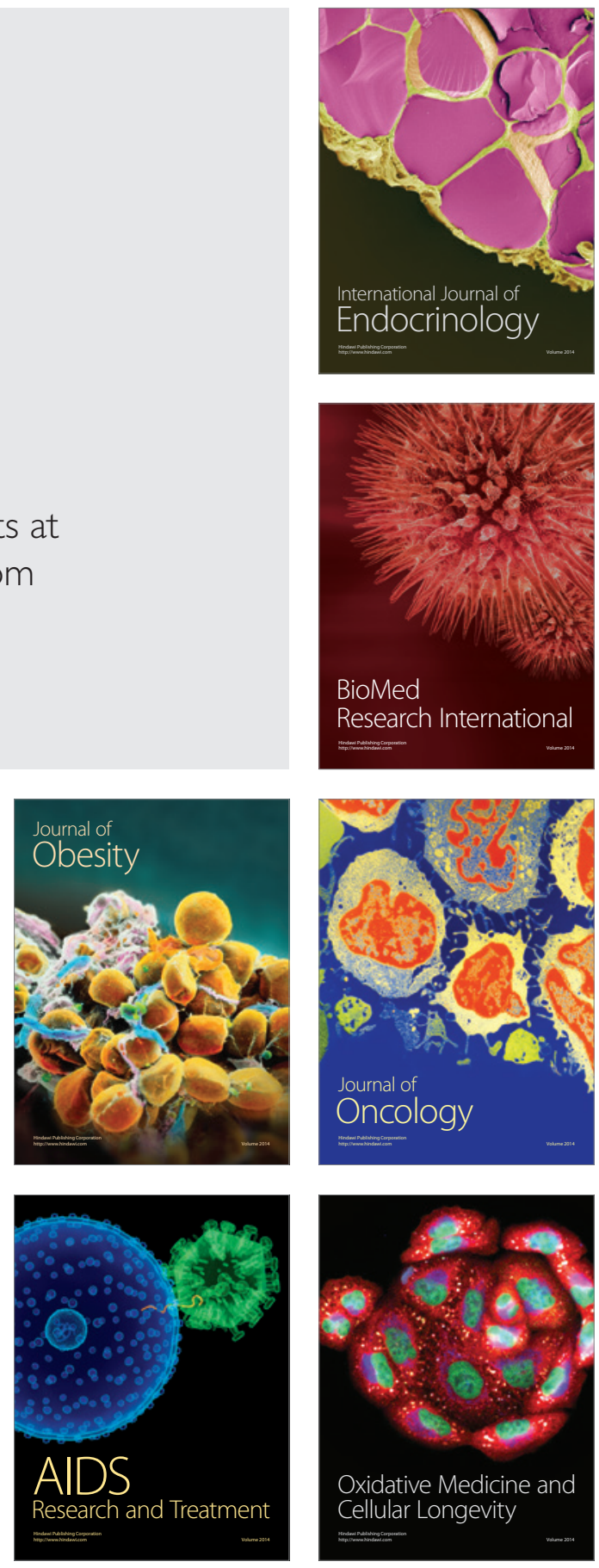